\title{
DESIGN PARAMETERS FOR THE DAMPED DETUNED ACCELERATING STRUCTURE*
}

\author{
K.Ko, K.Bane, R.Gluckstern, H.Hoag, N.Kroll, X.T.Lin, R.Miller, R.Ruth, K.Thompson, J.Wang, \\ Stanford Linear Accelerator Center, Stanford University, Stanford, CA 94309 USA, \\ University of California, San Diego, \\ University of Maryland, College Park, MD 20740
}

\begin{abstract}
The advanced accelerating cavities for the NLCTA (and anticipated for NLC) will incorporate damping as well as detuning. The damping is provided by a set of four waveguides (which also serve as pumping manifolds) that run parallel to the structure, with strong iris coupling to each cavity cell and terminated at each end by absorbers. The previously reported [1][2] equivalent circuit analysis has been refined and the dependence upon design parameters explored. We find that adequate damping can be provided by a single waveguide mode, leading to designs which are more compact than those initially considered. The design parameters and their rationale will be presented.
\end{abstract}

\section{Introduction}

The accelerating cavities for the NLC Test Accelerator (NLCTA) will incorporate damping as well as detuning (the DDS structure)[1][2]. The detuning suppresses the transverse wakefield experienced by closely trailing bunches, and the damping suppresses that experienced by the more remote bunches. The damping is provided by a set of four waveguides (which also serve as pumping manifolds) that run parallel to the structure, with strong iris coupling to each cavity cell and terminated at each end by absorbers. Absorption along the length of the manifold may also be provided. In the following we first review the underlying rationale of the manifold damping scheme and describe improvements in the analyses which have taken place since our last report[2]. This is followed by a description of our proposed design, its conceptual basis, and our current plans for its implementation.

\section{The manifold damping scheme}

In the detuned structure cell dimensions are varied along the length of the accelerator cavity in a gaussian manner so that the frequencies of the lower dipole mode of the individual cells vary over a range of approximately fifteen percent, and the frequencies of the resultant coupled modes (i.e., cavity modes; when not qualified, "mode" will be taken to mean "cavity mode") vary over a range of approximately $25 \%$. As shown in [3], these modes are localized standing waves with cell to cell amplitude variation which may be viewed as an effective wavelength.which varies smoothly

\footnotetext{
*Work supported by the Department of Energy, contracts DE-
} AC03-76SF00515. and extensively along the length of the mode. Their structure can be interpreted in the following manner. Each cell, if it were part of a periodic structure, would have associated with it a phase advance per cell (and hence a phase velocity) as a function of frequency. These quantities continue to have a significance in a localized sense when the cells are assembled to form a detuned structure. Thus we speak of a local phase advance ("per cell" is understood) and a local phase velocity. Modes terminate within the structure where the local phase advance is either zero or $\pi$, and approximately half the modes are entirely confined within the structure with phase advance $\pi$ at one end and zero at the other. A particular mode is excited by a velocity of light charge only if the range of local phase velocities associated with it includes that velocity. Similarly the mode couples strongly to a manifold mode only if the range also includes the phase velocity of the manifold mode. This implies that the coupling of a mode to a manifold mode is localized within the mode in the vicinity of the cell where the velocity match occurs.

Our theoretical analysis of these structures has so far been based on the single band equivalent circuit model discussed in [2]. There we derived the following equation for the damped cavity modes:

$$
\begin{aligned}
& \left(\frac{1}{f_{m}^{2}}-\frac{1}{f^{2}}\right) a_{m}+\frac{k_{m+\frac{1}{2}}}{2} a_{m+1}+\frac{k_{m-\frac{1}{2}}}{2} a_{m-1} \\
& =j \phi \frac{k}{k_{g}} \frac{Z_{0}}{Z} \sum_{n} \hat{k}_{m} \hat{k}_{n} \exp (-j \phi|n-m|) a_{n} .
\end{aligned}
$$

The LHS of Eq. (1) corresponds to the cell characteristics and the cell to cell coupling. Here $a_{m}$ is the excitation of cell $m, f_{m}$ its resonant frequency, $f$ the coupled mode frequency, and the $k_{m \pm 1 / 2}$ 's represent the coupling between adjacent cells. The RHS of Eq. (1) represents the coupling of the cells to one another via the manifold mode and their coupling to the matched loads which are assumed to terminate the manifold mode at each end. A summation over manifold modes (not shown explicitly to simplify the notation) is to be understood. Here $\hat{k}_{m}$ represents the coupling of the $m$ th cell to the manifold mode, $Z$ its wave impedence, $k_{g}$ its propagation constant, and $\phi=k_{g} P$ where $P$ is the period. As discussed in [2], Eq. (1) is a linear eigenvalue equation in $1 / f^{2}$ (with symmetric coefficients) when the RHS is neglected, and standard matrix diagonalization techniques can be used. The associated eigenvectors ( $\mathrm{col}-$ umn vectors in the $a_{m}$ ) may be used in conjunction with the RHS to evaluate, by means of standard perturbation 


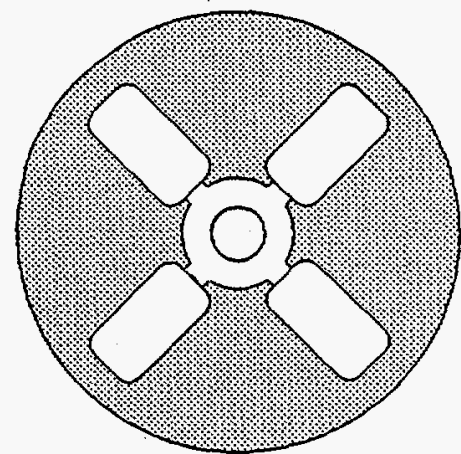

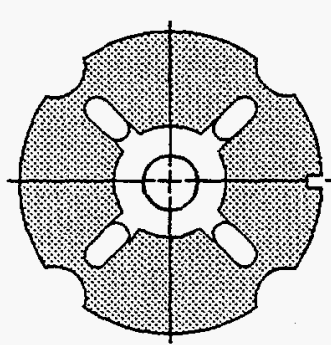

(b) (a)

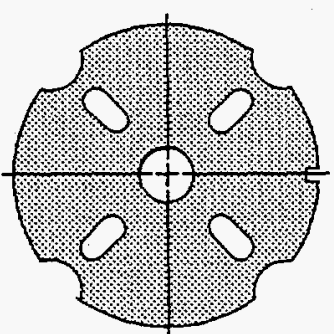

(c)

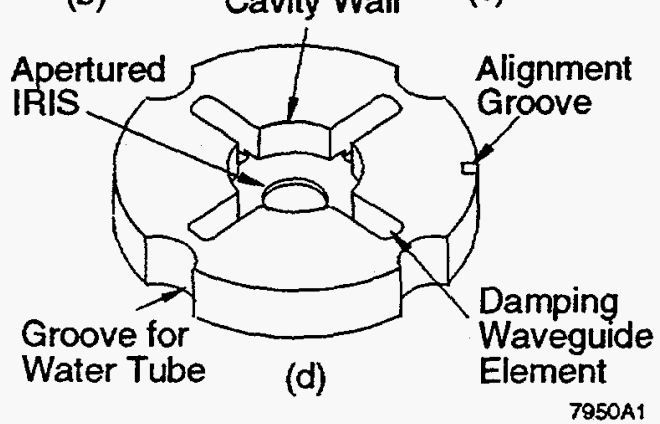

Figure. 1. (a) Cell-center cross section of multimode manifold, (b) cell-center cross section of single-mode manifold, (c) iris-centered cross section of single-mode manifold, (d) $3 \mathrm{D}$ view of single-mode manifold.

theory formulas, both the real and imaginary parts of the frequency shift due to the manifold coupling.

Because the matrix elements of the RHS are complex and involve the frequency in a complicated way, standard diagonalization techniques cannot be used to solve the full equation. Our procedure for determining the complex mode frequencies has been based upon the fact that the determinant of the coefficients of the $a_{m}$ must vanish in order to have a non-trivial solution. The mode frequencies for the undamped problem provide useful starting frequencies for an iterative procedure to find the roots of the determinant including damping. This approach has proved to be stable only when the change due to the RHS is sufficiently small. Alternating the iterative procedure with successive small increases in the coupling strength has, however, enabled us to overcome this limitation.

Once the frequencies have been determined it is staightforward to determine the mode vectors by means of Cramer's rule. We note that these vectors are also complex and while linearly independent, are no longer orthogonal. We have examined the space time behavior of a typical trapped mode making use of computer graphics. The previously localized portion still dominates the picture, but there is a small tail which extends over the entire length of the structure and represents the response of the cells to the field in the manifold. It has the character of an outgoing wave on both sides of the localization region with uniform wavelength (reflecting the fact that the manifold in this simulation was uniform). On the other hand, the ends of the localization region exhibit an ingoing wave character corresponding to the propagation of energy from the outer regions of the mode to the region where the mode couples well to the manifold. These wave propagation features can only occur for complex mode vectors.

The Bane-Gluckstern[3] procedure for relating these vectors to the wakefield requires some modification. The details can not be given here but we arrive at an analogue of their Eq. (2.48) which for us takes the form:

$$
\begin{gathered}
\tilde{V}(s) \approx 2 q_{e} x_{e} N L \sum_{p}\left[K_{p} \sin \left(2 \pi f_{p} s / c+\phi_{p}\right)\right. \\
\cdot \exp \left(-\pi f_{p} s / Q_{p}\right) \quad(s>0) .
\end{gathered}
$$

As compared to Bane and Gluckstern, our equation includes small manifold-induced corrections to the parameters, the small phase shifts $\phi_{p}$, and of course the damping terms. We have omitted a branch cut integral which corrects the behavior as $s$ goes to zero, becomes the dominant term for values of $s$ much larger than those of interest, but is believed to be negligible at $s$ values of interest.

We have compared the "exact" wakefield envelope function obtained using the iterative procedure with that obtained from perturbation theory, for a DDS with a single manifold mode having a $12 \mathrm{GHz}$ cutoff frequency and as large a coupling parameter as we are likely to use. The difference between the iterative and perturbative solutions is very small, and the coupling is strong enough to produce very satisfactory damping (see Ref. [2]). The $Q$ values are about 1000 . The shunt impedance degradation due to the manifolds is less than $3 \%$ over the first $2 / 3$ of the cells and rises to a maximum of about $5 \%$ at the end of the structure.

\section{The damped detuned structure design}

The manifold geometry discussed in references [1] and [2] is shown in midcell cross section in Fig. 2a. It has five propagating modes within the lower dipole band, the upper four of which contribute significantly to the damping. Because each of the manifold modes has its own dispersion characteristic, each of them couples to a particular cavity mode at a different cell. This is a desirable feature for strong coupling because distributing the damping along a cavity mode reduces the chance that its pattern will distort so that it decouples itself. As discussed above, however, the needed coupling is not strong enough to produce significant distortion even in the single mode case. Thus while calculations showed that the multimode manifold yielded very satisfactory damping with little shunt impedance degradation, it was decided to proceed with a single mode manifold design. 


\section{DISCLAIMER}

Portions of this document may be illegible in electronic image products. Images are produced from the best available original document. 
Cross sections of a typical element of the new structure taken at the center of a cell and at the center of the coupling iris are shown in Figs. $2 \mathrm{~b}$ and $2 c$, and a 3D view is shown in Fig. 2d. The new structure is seen to be both simpler and more compact than the multi manifold mode structure. MAFIA calculations show that the propagating manifold mode has a strong $\mathrm{TE}_{10}$ character. It has significant coupling only with the TE component of the dipole modes. We recall that the dipole modes are hybrid TETM modes, and that for the lower dipole mode the TE character is dominant at low phase advance, while the TM character dominates at large phase advance[4]. It is therefore necessary that the phase velocity match between the dipole mode and the manifold mode occur at low phase advance. We satisfy this requirement by adiabatically tapering the properties of the manifold so that the effective cutoff frequency of the manifold mode increases as one proceeds down the structure. Hence at any specified frequency the phase velocity of the manifold mode increases as one proceeds along the structure. Thus for the new structure the concept of local phase velocity applies both to the cavity mode and the manifold mode, and the coupling between the two occurs where these match.

We note that the manifold structure shown in Figs. 2b and $2 \mathrm{c}$ bears little resemblence to a waveguide, since $5 / 6$ of one of the narrow walls is removed for coupling to the accelerator cells. If, however one examines the mode spectrum and mode patterns in a zero phase advance MAFIA calclation, one observes a mode with a strong $\mathrm{TE}_{10}$ character well localized in the manifold, and we take its frequency as the manifold cutoff frequency. Furthermore the dependence of frequency on phase advance is, for small phase advance, quite similar to that of a waveguide with the cutoff frequency as defined above. The behavior in the vicinity of an avoided crossing is also very similar to the case of simple waveguide coupling and we have used the same methodology to determine the coupling strength to the manifold.

The general features of our initial design proposal, based upon the approach described above, are as follows: The thickness and beam hole dimensions of the coupling irises are taken to be the same as for the detuned structure already built and tested. The outer diameter of the cell slots $\left(L_{1}\right)$ varies from 2 to 1.5 inches, and their width is constant at 0.1968 inches. The slots in the coupling iris coincide with those of the smaller adjacent cell along width and outer circumference, while the inner diameter $\left(L_{2}\right)$ varies from 1.2 to .71 inches. Over the last third of the structure $L_{2}$ is less than the outer diameter of the associated cells. The cutoff frequency increases with decreasing $\left(L_{1}-L_{2}\right)$ and also with decreasing $L_{1}$. Over the entire structure it ranges from 12.1 to $14.0 \mathrm{GHz}$. The avoided crossing occurs at phase advance varying from 25 degrees to 82 degrees. As this phase advance increases, the cell to manifold coupling decreases. The decrease in $L_{2}$ has the opposite effect and was introduced to maintain the coupling strength. This remedy has the undesirable effect of decreasing the shunt impedence of the accelerating mode, so that it must be applied with circumspection. We note that there are a few modes for which the manifold is cutoff, and a substantial number for which it is cutoff at one end. The latter are presumably damped in an irregular manner due to the reflection from the cutoff end. None of the modes so affected intersect the light line so that their loss factor and their effect on the wakefield envelope function are expected to be small.

Once the manifold dimensions are fixed one must face the delicate task of determining the outer cell diameters $(2 b)$ so as to obtain an accurately tuned accelerating mode. This dimension has been accurately determined using a two dimensinal finite element program[5] for the detuned structure without manifolds. The dimensions obtained in this way are sufficiently accurate to permit construction with accurately machined parts without requiring subsequent hand tuning. The three dimensional structure with the manifolds is a more difficult computational challenge. We have used a MAFIA based method which aims at computing the difference in the cell diameter with and without slots. This difference, which ranges from approximately 20 to $30 \mathrm{mils}$, is then used to correct the previously determined accurate cell diameter. A fine triangular mesh which accurately traces circles of constant radius $r$ has been used. The computed radii are believed to be correct to within 0.1 mils, and the frequencies are expected to lie within $3 \mathrm{MHz}$ of the design value $(11.424 \mathrm{GHz})$. This would be close enough to allow hand tuning. As a check on our numerical methods and our evaluation of coupling parameters six samples each of cells number $10,70,106,156$, and 196 are being fabricated for cold testing.

Our estimate of the wakefield damping which the new structure will exhibit is based solely upon the rather crude modeling of [2]. It does not take account of the departure of the manifold from a simple waveguide, and the effect of the tapering on the modes which require damping has been inferred from the adiabatic principle without any explicit calculation. We have planned a theoretical program which deals with both of these deficiencies. Also we recognize that the specification that the manifolds be terminated by matched loads in their propagation range may be difficult to realize in practice. Some theoretical effort will be devoted to the design of load structures, and the possibility of distributed loading along the manifold with lossy materials will be explored.

\section{References}

[1] N.Kroll, et.al., 17th Int'l Linear Accel. Conf., Tsukuba, Japan, 21-26 August 1994.

[2] N.Kroll, et.al., 6th Workshop on Advanced Accelerator Concepts, Lake Geneva, WI, 12-18 June 1994; SLACPUB-6660.

[3] K.L.F. Bane and R.L. Gluckstern, Part. Accel. 42, 123 (1993); SLAC-PUB-5783.

[4] J.W. Wang and G.A. Loew, 1990 Linear Accel. Conference, Albuquerque, NM, 10-14 Sept. 1990; SLAC-PUB5321.

[5] J.W. Wang and E.M. Nelson, 1993 Part. Accel. Conf., Washington, DC, 17-20 May 1993; SLAC-PUB-6142. 


\section{DISCLAIMER}

This report was prepared as an account of work sponsored by an agency of the United States Government. Neither the United States Government nor any agency thereof, nor any of their employees, makes any warranty, express or implied, or assumes any legal liability or responsibility for the accuracy, completeness; or usefulness of any information, apparatus, product, or process disclosed, or represents that its use would not infringe privately owned rights. Reference herein to any specific commercial product, process, or service by trade name, trademark, manufacturer, or otherwise does not necessarily constitute or imply its endorsement, recommendation, or favoring by the United States Government or any agency thereof. The views and opinions of authors expressed herein do not necessarily state or reflect those of the United States Government or any agency thereof. 